\title{
ON A PENROSE INEQUALITY WITH CHARGE
}

\author{
GILBERT WEINSTEIN AND SUMIO YAMADA
}

\begin{abstract}
We construct a time-symmetric asymptotically flat initial data set to the Einstein-Maxwell Equations which satisfies

$$
m-\frac{1}{2}\left(R+\frac{Q^{2}}{R}\right)<0
$$

where $m$ is the total mass, $R=\sqrt{A / 4 \pi}$ is the area radius of the outermost horizon and $Q$ is the total charge. This yields a counter-example to a natural extension of the Penrose Inequality for charged black holes.
\end{abstract}

\section{INTRODUCTION}

There has recently been much interest among geometers and mathematical relativists in inequalities bounding the total mass of initial data sets from below in terms of other geometrical quantities. The first such inequality is the Positive Mass Theorem [12, 14. We rephrase the Riemannian version of this result as the following variational statement: among all time-symmetric asymptotically flat initial data sets for the Einstein-Vacuum Equations, flat Euclidean 3-space is the unique minimizer of the total mass. Thus, the total mass satisfies $m \geqslant 0$ with equality if and only if the data set is isometric to $\mathbb{R}^{3}$ with the flat metric. See the next section for precise definitions.

A stronger result is the Riemannian version of the Penrose Inequality, which can be stated in a similar variational vein: among all time-symmetric asymptotically flat initial data sets for the Einstein-Vacuum Equations with an outermost minimal surface of area $A$, the Schwarzschild slice is the unique minimizer of the total mass. In other words, $m \geqslant R / 2$ where $R=\sqrt{A / 4 \pi}$ is the area radius of the outermost horizon, and equality occurs if and only if the data is isometric to the Schwarzschild slice:

$$
g_{i j}=\left(1+\frac{m}{2 r}\right)^{4} \delta_{i j}
$$

When these results are phrased in this fashion, a natural question is whether similar variational characterizations of the other known stationary solutions of the Einstein Equations hold. In particular, one could ask whether among all asymptotically flat axisymmetric maximal gauge initial data sets for the Einstein-Vacuum Equations with an outermost minimal

Date: June 8, 2021.

1991 Mathematics Subject Classification. 58J05.

The research of the first author was supported in part by NSF Grant DMS-0205545. The research of the second author was supported in part by NSF Grant DMS-0222387. 
surface of area $A$ and angular momentum $J$, the Kerr slice is the unique minimizer of the mass. Such a statement would imply that:

$$
m \geqslant \frac{1}{2}\left(R^{2}+\frac{4 J^{2}}{R^{2}}\right)^{1 / 2} .
$$

with equality if and only if the data is isometric to the Kerr slice. Since it is not known how to define the angular momentum of a finite surface, it is necessary to assume the axisymmetry of the data set. With that hypothesis, if $X$ is the generator of the axisymmetry, then the Komar integral:

$$
J(S)=\frac{1}{8 \pi} \int_{S} k_{i j} X^{i} n^{j} d A
$$

gives a quantity which depends only on the homological type of $S$ and tends to the total angular momentum, as $S$ tends to the sphere at infinity.

A similar question can be asked with charge replacing angular momentum: is the Reissner-Nordström slice the unique minimizer of the mass among all asymptotically flat time-symmetric initial data sets for the Einstein-Maxwell Equations? This is equivalent to asking whether the following inequality holds for any data set:

$$
m \geqslant \frac{1}{2}\left(R+\frac{Q^{2}}{R}\right)
$$

where $Q$ is the total charge, with equality if and only if the data is a ReissnerNordström slice. As above, the charge:

$$
Q(S)=\frac{1}{4 \pi} \int_{S} E_{i} n^{i} d A
$$

depends only on the homological type of $S$.

When the horizon is connected, inequality (2) can be proved by using the Inverse Mean Curvature flow [6, 9]. Indeed, the argument in [9] relies simply on Geroch montonicity of the Hawking mass - which still holds for the weak flow introduced by Huisken and Ilmanen in 6 - while keeping track of the scalar curvature term $R_{g}=2\left(|E|^{2}+|B|^{2}\right)$. However, when the horizon has several components the same argument yields only the following inequality:

$$
m \geqslant \frac{1}{2} \max _{i}\left(R_{i}+\frac{\left(\min \sum_{i} \varepsilon_{i} Q_{i}\right)^{2}}{R_{i}}\right),
$$

where $R_{i}$ and $Q_{i}$ are the area radii and charges of the components of the horizon $i=1, \ldots, N, \varepsilon_{i}=0$ or 1 , and the minimum is taken over all possible combinations.

It is the purpose of this paper to point out that (2) does not hold. We prove: 
Theorem 1. There is a strongly asymptotically flat time-symmetric initial data set $(M, g, E, 0)$ for the Einstein-Maxwell Equations such that:

$$
m-\frac{1}{2}\left(R+\frac{Q^{2}}{R}\right)<0 .
$$

In 1984, Gibbons 4] conjectured an inequality similar to (2). However, in his conjecture, the right hand side of (2) is taken to be additive over connected components of the horizon. Thus, Gibbons's conjecture states that:

$$
m \geqslant \frac{1}{2} \sum_{i}\left(R_{i}+\frac{Q_{i}^{2}}{R_{i}}\right) .
$$

In particular, when there is no electromagnetic field this inequality reduces to:

$$
m \geqslant \frac{1}{2} \sum_{i} R_{i}
$$

which is stronger than the usual Riemannian Penrose inequality;

$$
m \geqslant \frac{1}{2}\left(\sum_{i} R_{i}^{2}\right)^{1 / 2} .
$$

It is not known whether (5) holds, but two Schwarzschild slices a large distance apart would seem to violate this inequality. Gibbons further conjectured that equality occurs in (4) if and only if the data is MajumdarPapapetrou; see the next section for a description of these metrics. We note that these metrics do not actually have horizons and are not asymptotically flat in the sense of Definition 11. Instead, they have one asymptotically flat end and $N$ asymptotically cylindrical ends which we will call necks. The cross-sections of these necks are spheres with mean curvature tending to zero as the surfaces goes further down the end.

Our construction is based on the fact that the Majumdar-Papapetrou metrics 'violate' (2), say with $N=2$, and $m_{1}=m_{2}$. They do not strictly speaking violate (2) since they are not asymptotically flat and do not possess horizons. In order to remedy these failures, we glue two such copies along the necks. The gluing procedure we use is an adaptation of the conformal perturbation method developed for the vacuum case in [7. In fact in our setting, some of the technical difficulties arising from the generality of the construction in [7] are absent. However, while it is easy to show the existence of a two-component minimal surface in the resulting metric, we must also show that (2) is violated with $R$ the area radius of the outermost horizon. This requires ruling out minimal surfaces outside the necks which we can accomplish by letting $m \rightarrow 0$ which is equivalent after rescaling to taking the two masses in the initial Majumdar-Papapetrou far apart.

We point out that this counter-example has little to do with the Cosmic Censorship conjecture. In fact, as pointed out by Jang [9], inequality (2) is 
equivalent to:

$$
m-\sqrt{m^{2}-Q^{2}} \leqslant R \leqslant m+\sqrt{m^{2}-Q^{2}},
$$

and only the upper bound would follow from Cosmic Censorship using Penrose's heuristic argument. Our counter-example violates the lower bound.

The paper is organized as follows. In the next section, we define some terms, and set-up the notation. In section 3, we carry out the gluing. In the last section, we show that the parameters can be chosen so that the resulting initial data violates (2)

We wish to thank Greg Galloway, Robert Hardt, and Robert Wald for useful discussions on this paper. We thank the American Institute of Mathematics for its hospitality. The first author also thanks the Erwin Schrödinger Institute for its hospitality.

\section{Preliminaries}

Definition 1. Let $(M, g)$ be a 3-dimensional Riemannian manifold. We say that $(M, g)$ is strongly asymptotically flat (SAF) if there is a compact set $K \subset M$ such that $M \backslash K$ is the disjoint union of finitely many ends $N_{\nu}$, $\nu=1, \ldots, k$, each end $N_{\nu}$ is diffeomorphic to $\mathbb{R}^{3}$ minus a ball and admits a coordinate system in which the metric satisfies:

$$
g_{i j}-\delta_{i j} \in C_{-1}^{2, \alpha}\left(N_{\nu}\right) \text {. }
$$

Here $C_{-1}^{2, \alpha}\left(N_{\nu}\right)$ denotes the class of functions $\phi$ such that $r|\phi|, r^{2}|\partial \phi|$, $r^{3}\left|\partial^{2} \phi\right|$ and $r^{3+\alpha}|x-y|^{-\alpha}\left|\partial^{2} \phi(x)-\partial^{2} \phi(y)\right|$ are bounded. While the bound is coordinate dependent, the set of function is independent of coordinates. We will focus out attention on one end which we will denote by $N_{+}$. We will denote all the other ends collectively as $N_{-}$. In fact, in this paper we are dealing exclusively with two-ended SAF manifolds so that $N_{-}$consists of only one end. By adding a point $\infty_{-}$(or in the general case $k-1$ points) at infinity in $N_{-}$and conformally compactifying, we obtain an asymptotically flat Riemannian manifold with one end. We now consider the class $\mathcal{S}$ of smooth surfaces $S$ which bound a compact region $\Omega$ such that $\infty_{-} \in \Omega$. In this class, it makes sense to speak of the outer unit normal. If $S_{1}, S_{2} \in \mathcal{S}$, we will say that $S_{1}$ encloses $S_{2}$ if the corresponding regions $\Omega_{1}$ and $\Omega_{2}$ satisfy $\Omega_{1} \supset \Omega_{2}$.

If $(M, g)$ is strongly asymptotically flat, the total mass $m$ of the end $N_{+}$ is defined by:

$$
m=\frac{1}{16 \pi} \lim _{r \rightarrow \infty} \int_{S_{r}}\left(g_{i j, j}-g_{j j, i}\right) n^{i} d A,
$$

where $S_{r}$ is the Euclidean coordinate sphere in $N_{+}, n$ its unit normal in $\delta$, and $d A$ the area element induced on $S_{r}$ from $\delta$.

Definition 2. A horizon $S$ is a minimal surface in $(M, g)$ which belongs to $\mathcal{S}$. An outermost horizon is a horizon which is not enclosed within any 
other horizon. A surface $S \in \mathcal{S}$ is outer minimizing if it has area no greater than any other surface which encloses it.

Note that for $r$ large enough, $S_{r} \in \mathcal{S}$ and has positive mean curvature with respect to its outer unit normal. Thus, by minimizing area over all surfaces in $\mathcal{S}$ which enclose the outermost horizon $S$, and are enclosed in $S_{r}$, we obtain a minimal surface $S_{1}$ which encloses $S$. It then follows from the outermost property of $S$ that $S=S_{1}$; see [11, Theorem 1', p. 645]. We conclude that an outermost horizon is also outer minimizing, a fact which will be used in the last section.

A time-symmetric initial data set $(M, g, E, B)$ for the Einstein-Maxwell Equations consists of a Riemannian manifold $(M, g)$, and two vector fields $E$ and $B$ on $M$ such that:

$$
R_{g}=2\left(|E|_{g}^{2}+|B|_{g}^{2}\right), \operatorname{div}_{g} E=\operatorname{div}_{g} B=0, E \times B=0, \int_{S} g\left(B, n_{g}\right) d A=0,
$$

where $R_{g}$ is the scalar curvature of $g$, and $S \subset M$ is an arbitrary closed surface with normal $n_{g}$ of unit length in $g$. We say that the set $(M, g, E, B)$ is strongly asymptotically flat if $(M, g)$ is $\mathrm{SAF}$, and if $E \in C_{-2}^{2, \alpha}, B \in C_{-3}^{2, \alpha}$.

Choose $N>0, m_{k}>0$, and $p_{k} \in \mathbb{R}^{3}$ for $k=1, \ldots, N$, and let $r_{k}$ denote the Euclidean distance to $p_{k}$ in $\mathbb{R}^{3}$. The Majumdar-Papapetrou solutions are given by:

$$
u=\left(1+\sum_{k=1}^{N} \frac{m_{k}}{r_{k}}\right)^{1 / 2}, \quad g_{i j}=u^{4} \delta_{i j}, \quad E_{i}=2 \nabla_{i} \log u, \quad B_{i}=0 .
$$

When $N=1$, this is simply the extreme case $m=|Q|$ of the ReissnerNordström data set. Note that if we take $E_{-}=-2 \nabla \log u$ instead of $E=$ $2 \nabla \log u$, we get another solution with charges of opposite sign.

For simplicity, we will restrict ourselves to the case $N=2, m_{1}=m_{2}=m$, i.e. $u=\left(1+m / r_{1}+m / r_{2}\right)^{1 / 2}$. It is not difficult to check that $(M, g, E, 0)$ satisfies the Einstein-Maxwell time-symmetric constraints. In fact, the metric $-u^{-4} d t^{2}+g$ is a static solution of the Einstein-Maxwell equations. Let $r$ denote the Euclidean distance from the origin. We denote by $B_{i}(\rho)=\left\{r_{i}<\rho\right\}$ the Euclidean ball of radius $\rho$ centered at $p_{i}$, and by $B_{0}(\rho)=\{r<\rho\}$ the Euclidean ball of radius $\rho$ centered at the origin. Note that for $R$ large enough $N=\mathbb{R}^{3} \backslash B_{0}(R)$ equipped with the metric $g$ is a SAF end, and the necks $B_{i}(\rho) \backslash\left\{p_{i}\right\}$ are asymptotically cylindrical. It is easy to check that the total mass $\mu$ of $N$ is $2 m$, the total charge $Q=\int_{S} g(E, n) d A$ is $2 m$, while the total cross sectional area $A$ of both necks is asymptotically $8 \pi \mathrm{m}^{2}$, i.e., $R=\sqrt{2} m$. Thus, we get:

$$
\mu-\frac{1}{2}\left(R+\frac{Q^{2}}{R}\right)=2 m-\frac{1}{2}(\sqrt{2} m+2 \sqrt{2} m)=m\left(2-\frac{3}{\sqrt{2}}\right)<0 .
$$

However $(M, g)$ admits no horizon. In the next section, we remedy this by gluing at the necks a second copy of opposite charges. The solution of the 
constraints is achieved through a conformal perturbation argument. We will then show in Section 4 that the resulting data set possesses a horizon which violates (2).

\section{The Gluing}

Let $\left(M_{ \pm}, g_{ \pm}, E_{ \pm}, 0\right)$ be two copies of the Majumdar-Papapetrou data, with $E_{-}=-E_{+}$. In this section, we show that we can glue these two copies along their necks. This gluing will be performed by a perturbation method with perturbation parameter $T>0$ large. Whenever a possible ambiguity might arise, we use a subscript (or superscript) + (or - respectively) to indicate a quantity associated with $M_{+}$(or $M_{-}$respectively).

For convenience, we take $p_{1}=(0,0,1)$ and $p_{2}=(0,0,-1)$. The gluing is accomplished in three steps. In the first step, we truncate the necks at $r_{i}=e^{-T}$, and introduce cut-offs in the regions $e^{-T+1}<r_{i}^{ \pm}<e^{-T+2}$ to obtain a transition to round cylinders. This yields data on $M_{+}$and $M_{-}$ which matches in the regions $e^{-T}<r_{i}^{ \pm}<e^{-T+1}$ of the necks. We can then identify the corresponding boundaries $r_{i}^{ \pm}=e^{-T}$ in $M_{+}$and $M_{-}$creating a two-ended SAF data set $(\hat{M}, \hat{g}, \hat{E}, 0)$. However, this data no longer satisfies the constraint equations in the cut-off regions. In the second step, we restore the divergence constraint $\operatorname{div}_{\hat{g}} \hat{E}^{\prime}=0, \hat{E}^{\prime}=\hat{E}-\nabla \varphi$, by solving a linear problem $\Delta_{\hat{g}} \varphi=\operatorname{div}_{\hat{g}} \hat{E}, \varphi \rightarrow 0$ at $\infty$. Finally, in the last step, we use a perturbation argument to find a conformal deformation $(\widetilde{M}, \tilde{g}, \widetilde{E}, 0)=$ $\left(\hat{M}, \phi^{4} \hat{g}, \phi^{-6} \hat{E}^{\prime}\right)$ which satisfies the constraints. It is easy to see that the divergence constraint is automatically preserved under the above conformal transformation $g \mapsto \phi^{4} g, \hat{E}^{\prime} \mapsto \phi^{-6} \hat{E}^{\prime}$, i.e., we have $\operatorname{div}_{\tilde{g}} \widetilde{E}=0$. The Gauss constraint $R_{\tilde{g}}=|\widetilde{E}|_{\tilde{g}}^{2}$ is then satisfied if and only if $\phi$ satisfies the following nonlinear equation [8]:

$$
L_{\hat{g}} \phi=-\frac{\left|\hat{E}^{\prime}\right|_{\hat{g}}^{2}}{4 \phi^{3}}
$$

where $L_{\hat{g}}=\Delta_{\hat{g}}-\frac{1}{8} R_{\hat{g}}$ is the conformal Laplacian of $\hat{g}$. Section 3.3 is therefore devoted to showing that for $T$ large enough, there is a positive solution $\phi$ of (17) such that $\phi-1$ is small in $C_{-1}^{2, \alpha}$. This gluing technique is an adaptation of 7 .

3.0. Function Spaces and Elliptic Theory. Let $(M, g)$ be a SAF manifold with $K \subset M$ compact and $M \backslash K$ the disjoint union of finitely many ends $N_{\nu}$. Let $\sigma \geqslant 1$ be a weight function on $M$ such that $\sigma=1$ on $K$, and equals the Euclidean distance $r$ on each end $N_{\nu}$ for $r$ large enough. Let $C_{-\beta}^{k, \alpha}(M)$ be the set of functions $\phi$ on $M$ whose $k$-th order derivatives are 
Hölder continuous and for which the norm $\|\phi\|_{C_{-\beta}^{k, \alpha}}$ defined below is finite:

$$
\begin{aligned}
\|\phi\|_{C_{-\beta}^{k}} & =\sum_{i=0}^{k}\left\|\sigma^{\beta+i} D^{i} \phi\right\|_{C^{0}} \\
{\left[D^{k} \phi\right]_{\alpha,-\beta} } & =\sup _{0<\operatorname{dist}(x, y)<\rho} \sigma(x, y)^{\beta+\alpha} \frac{\left|P_{y}^{x} D^{k} \phi(y)-D^{k} \phi(x)\right|}{\operatorname{dist}(x, y)^{\alpha}} \\
\|\phi\|_{C_{-\beta}^{k, \alpha}} & =\|\phi\|_{C_{-\beta}^{k}}+\left[D^{k} \phi\right]_{C_{-\beta-k}^{\alpha}} .
\end{aligned}
$$

Here $D^{i} \phi$ represents the tensor of $i$-th order derivatives of $\phi, \rho$ is the injectivity radius of $(M, g), \sigma(x, y)=\max \{\sigma(x), \sigma(y)\}$, and $P_{y}^{x}$ is parallel translation along the shortest geodesic from $y$ to $x$.

Theorem 2. Let $(M, g)$ be a SAF manifold.

(a) Let $\phi \in C_{-\beta}^{0}(M)$ and $\Delta_{g} \phi \in C_{-\beta-2}^{0, \alpha}(M)$, then $\phi \in C_{-\beta}^{2, \alpha}(M)$ and

$$
\|\phi\|_{C_{-\beta}^{2, \alpha}} \leqslant C\left(\|\phi\|_{C_{-\beta}^{0}}+\left\|\Delta_{g} \phi\right\|_{C_{-\beta-2}^{0, \alpha}}\right) .
$$

(b) Let $0<\beta<1, \nu>2$, and let $h \in C_{-\nu}^{0, \alpha}(M)$. If the operator $\Delta_{g}-h$ : $C_{-\beta}^{2, \alpha}(M) \rightarrow C_{-\beta-2}^{0, \alpha}(M)$ is injective then it is an isomorphism.

This theorem is stated in [10, but the reader is referred to [3] for the proof. Unfortunately, the proof of part (b) in [3] has a small gap, which is nevertheless easily remedied. For details, please refer to [13, Appendix], where a complete proof is given for the case $M=\mathbb{R}^{3}$. The proof for general $\mathrm{SAF}$ manifolds is a straightforward combination of the arguments in 3] and [13].

3.1. Preparation. Fix $T>0$, and let $\chi(r)$ be a smooth positive nondecreasing cut-off function such that $\chi(r)=1$ for $r>e^{-T+2}$, and $\chi(r)=0$ for $r<e^{-T+1}$. Let $\chi_{i}=\chi\left(r_{i}\right), i=1,2$, and define:

$$
\hat{u}=\left(\chi_{1} \chi_{2}+\chi_{2} \frac{m}{r_{1}}+\chi_{1} \frac{m}{r_{2}}\right)^{1 / 2}, \quad \hat{g}=\hat{u}^{4} \delta=\left(\frac{\hat{u}}{u}\right)^{4} g, \quad \hat{E}_{i}=2 \nabla_{i} \log \hat{u} .
$$

We note that:

$$
\left|1-(\hat{u} / u)^{4}\right| \leqslant C e^{-T}, \quad|\nabla \log \hat{u}-\nabla \log u|_{g} \leqslant C e^{-T}, \quad\left|\Delta_{g}(\hat{u} / u)\right| \leqslant C e^{-T}
$$

where $C$ is a constant independent of $T$. This implies

$$
|\hat{g}-g|_{g} \leqslant C e^{-T},\left.\quad\left|R_{\hat{g}}-2\right| \hat{E}\right|_{\hat{g}} ^{2}\left|\leqslant C e^{-T}, \quad\right| \operatorname{div}_{\hat{g}} \hat{E} \mid \leqslant C e^{-T} .
$$

Introduce the notations:

$$
\begin{gathered}
B_{i}(\rho)=\left\{r_{i}<\rho\right\}, \quad D(\rho)=B_{1}(\rho) \cup B_{2}(\rho) \\
\Gamma_{i}(\rho)=\left\{e^{-T} \leqslant r_{i}<\rho\right\}, \quad \Gamma(\rho)=\Gamma_{1}(\rho) \cup \Gamma_{2}(\rho) .
\end{gathered}
$$


On $\Gamma_{i}\left(e^{-T+1}\right), \hat{g}=m^{2}\left(d r_{i}^{2} / r_{i}^{2}+d \omega^{2}\right)$ is a round cylindrical metric with $d \omega^{2}$ the standard metric on the unit sphere, and $\hat{E}=d r_{i} / r_{i}$ is parallel. Thus, if we take two copies $M_{ \pm}=\mathbb{R}^{3} \backslash D\left(e^{-T}\right)$, then both the metrics $\hat{g}_{ \pm}=\hat{g}$ and the vector fields $\hat{E}_{ \pm}= \pm \hat{E}$ match on $r_{i}^{ \pm}=e^{-T}$, and we can identify these boundaries to form a doubly-connected-sum $\hat{M}=M_{+} \# M_{-}$. We will denote the metric on $\hat{M}$ by $\hat{g}$ and the vector field by $\hat{E}$. We note that $(\hat{M}, \hat{g})$ is a two-ended SAF manifold. We denote $\hat{\Gamma}_{i}(\rho)=\Gamma_{i}^{+}(\rho) \cup \Gamma_{i}^{-}(\rho)$ and $\hat{\Gamma}(\rho)=\Gamma^{+}(\rho) \cup \Gamma^{-}(\rho)$. We have suppressed the dependence on $T$ in order not to encumber the notation.

We now fix the weight function $\sigma=\sigma(r)$ to be 1 on $\left\{r^{ \pm} \leqslant 3\right\}$ in $M_{ \pm}$, monotone in $r$, and equal to $r^{ \pm}$on $\left\{r^{ \pm}>4\right\}$. In addition, we can assume that it is even with respect to reflections across the cuts $\partial M_{+}=\left\{r_{1}=\right.$ $\left.e^{-T}\right\} \cup\left\{r_{2}=r^{-T}\right\}$. Note that since $\hat{u} / u=1$ outside $\hat{\Gamma}\left(e^{-T+2}\right)$, the quantities in (10) vanish outside this set, hence these estimates hold also with any weighted norms. In particular:

$$
\left\|R_{\hat{g}}-2|\hat{E}|_{\hat{g}}^{2}\right\|_{C_{-3}^{0, \alpha}} \leqslant C e^{-T}, \quad\left\|\operatorname{div}_{\hat{g}} \hat{E}\right\|_{C_{-3}^{0, \alpha}} \leqslant C e^{-T}
$$

Throughout the rest of this section, $C, C^{\prime}, c$ will denote various constants independent of $T$. In order to simplify the notation, we may at times change the value of such constants. This abuse of notation can be justified by simply taking the maximum of the previous and current value of the constant.

We will need the following essentially local elliptic estimate.

Proposition 1. Let $0<\beta<1, \nu>2$, and let $h \in C_{-\nu}^{0, \alpha}$ satisfy $h \geqslant 0$. There is a constant $C$ independent of $T$, such that for each $T$ large enough

$$
\|\phi\|_{C_{-\beta}^{2, \alpha}} \leqslant C\left(\|\phi\|_{C_{-\beta}^{0}}+\left\|\left(\Delta_{\hat{g}}-h\right) \phi\right\|_{C_{-\beta-2}^{0, \alpha}}\right) .
$$

Proof. On either end $\Omega_{ \pm}=M_{ \pm} \backslash B_{0}^{ \pm}(4)$, we can use an argument using local estimates and the scaling of annuli as in [13, Proposition 26] to get a weighted estimate:

$$
\|\phi\|_{C_{-\beta}^{2, \alpha}\left(\Omega_{ \pm}\right)} \leqslant C\left(\|\phi\|_{C_{-\beta}^{0}\left(\Omega_{ \pm}^{\prime}\right)}+\left\|\left(\Delta_{\hat{g}}-h\right) \phi\right\|_{C_{-\beta-2}^{0, \alpha}\left(\Omega_{ \pm}^{\prime}\right)}\right) .
$$

with a constant $C$ independent of $T$, where $\Omega_{ \pm}^{\prime}=M_{ \pm} \backslash B_{0}^{ \pm}(3)$. Now, let $K=\hat{M} \backslash\left[\left\{r^{+} \geqslant 5\right\} \cup\left\{r^{-} \geqslant 5\right\}\right]$, then $K$ can be covered by finitely many geodesic balls $B_{q_{i}}^{\hat{g}}(\rho)$ of radius $\rho>0$ sufficiently small, so that the elliptic constant of $\hat{g}$ written in normal coordinates on $B_{q_{i}}^{\hat{g}}(2 \rho)$ is uniformly bounded above and below. While the number of balls depends on $T, \rho$ can be chosen independently of $T$. We have local elliptic estimates:

$$
\|\phi\|_{C^{2, \alpha}\left(B_{q_{i}}^{\hat{g}}(\rho)\right)} \leqslant C\left(\|\phi\|_{C^{0}\left(B_{q_{i}}^{\hat{g}}(2 \rho)\right)}+\left\|\left(\Delta_{\hat{g}}-h\right) \phi\right\|_{C^{0, \alpha}\left(B_{q_{i}}^{\hat{g}}(2 \rho)\right)}\right),
$$

where $C$ depends on $\rho$ but is independent of $i$ or $T$. Collecting these estimates yields (14). 
3.2. The Divergence Constraint. In this section, we restore the divergence constraint by solving the following linear problem:

$$
\Delta_{\hat{g}} \varphi=f, \quad \varphi \rightarrow 0 \text { at } \infty .
$$

where $f=\operatorname{div}_{\hat{g}}(\hat{E})$. We must also ensure that $\varphi$ tends to zero when $T$ tends to infinity.

Proposition 2. For each $m>0$ small enough, and each $T$ large enough, there is a unique solution $\varphi \in C_{-1}^{2, \alpha}$ of the equation:

$$
\Delta_{\hat{g}} \varphi=f
$$

on $\hat{M}$, where $f=\operatorname{div}_{\hat{g}}(\hat{E})$. Furthermore,

$$
\|\varphi\|_{C_{-1}^{2, \alpha}} \leqslant C T^{2} e^{-T}
$$

where the constant $C$ is independent of $T$.

Proof. The existence of a solution $\varphi \in C_{-1}^{2, \alpha}$ is standard, see e.g. 3]. The smallness of $\varphi$, inequality (15), will follow from the elliptic estimates in Proposition 1 once we obtain a weighted supremum bound:

$$
\sup _{\hat{M}} \sigma|\varphi| \leqslant C T^{2} e^{-T},
$$

where $C$ is independent of $T$. This is obtained by a comparison argument using the maximum principle. Note that the function $f$ has $\operatorname{supp} f \subset$ $\hat{\Gamma}\left(e^{-T+2}\right)$, and $P=\sup _{T}\left(e^{T} \sup |f|\right)<\infty$. Furthermore, $f$ is odd with respect to reflection across the cuts $\partial M_{+}$which implies that $\varphi$ is also odd, hence $\varphi=0$ on $\partial M_{+}$. Now let:

$$
\psi(r)=-e^{-T} \int_{e^{-T}}^{r} \frac{\log (s)}{s(s+m)} d s .
$$

We claim that if $m$ is small enough and $T$ is large enough, then $w=\psi\left(r_{1}\right)+$ $\psi\left(r_{2}\right)$ has the following properties on $M_{+}$:

(i) $0<w \leqslant m^{-1} T^{2} e^{-T}$ on $M_{+}$.

(ii) $\Delta_{\hat{g}} w \leqslant 0$ on $M_{+}$.

(iii) $\Delta_{\hat{g}} w \leqslant-c e^{-T}$ on $\Gamma\left(e^{-T+2}\right)$ for some $c>0$ independent of $T$.

These properties imply that $\varphi-c^{-1} P w$ satisfies $\Delta_{\hat{g}}\left(\varphi-c^{-1} P w\right) \geqslant 0$, and $\varphi-c^{-1} P w \leqslant 0$ both on $\partial M_{+}$and at $\infty$. Thus, we get $\varphi \leqslant c^{-1} P w$ on $M_{+}$. Similarly, by considering the function $\varphi+c^{-1} P w$, we get $\varphi \geqslant-c^{-1} P w$ on $M_{+}$. This yields an unweighted supremum estimate:

$$
\sup _{M_{+}}|\varphi| \leqslant \frac{P}{c m} T^{2} e^{-T}
$$

By symmetry, the same estimate holds on $M_{-}$. Now, in order to get the weighted estimate (16), let $\Omega=M_{+} \backslash B_{0}^{+}(3)$, and let $v$ be the solution of the following problem:

$$
\Delta_{g} v=0 \text { in } \Omega, \quad v=1 \text { on } \partial \Omega, \quad v \rightarrow 0 \text { at } \infty .
$$


There is a constant $C$ such that $0<v \leqslant C \sigma^{-1}$. Let $K=P / \mathrm{cm}$, then the functions $\pm \varphi+K T^{2} e^{-T} v$ are harmonic in $\Omega$ with respect to $g=\hat{g}$, are non-negative on $\partial \Omega$, and tends to 0 at $\infty$, hence by the maximum principle $\pm \varphi+K T^{2} e^{-T} v \geqslant 0$ in $\Omega$. Hence, we obtain $\sigma|\varphi| \leqslant K C T^{2} e^{-T}$ on $\Omega$. Combining with (17), the weighted estimate (16) follows.

It remains to prove the claims (i)-(iii). Denote $\psi_{i}=\psi\left(r_{i}\right)$, and note that

$$
\max \psi_{i}=\psi(1)=-e^{-T} \int_{e^{-T}}^{1} \frac{\log s}{s(s+m)} d s \leqslant \frac{1}{2 m} T^{2} e^{-T},
$$

whence $w \leqslant m^{-1} T^{2} e^{-T}$. A similar estimate shows that $\psi(1)>T^{2} e^{-T} / 4 m$. On the other hand

$$
\psi(1)-\psi(\infty)=e^{-T} \int_{1}^{\infty} \frac{\log s}{s(s+m)} d s \leqslant e^{-T}<\psi(1),
$$

provided $T$ is large enough. We conclude that $w>0$ if $T$ is large enough proving (i). In order to establish (ii) and (iii), we first note that it is sufficient to prove these with $\hat{g}$ replaced by $g$. Indeed, suppose that (i) and (ii) hold with $g$ instead of $\hat{g}$. Then we have:

$$
\Delta_{\hat{g}} w=\left(\frac{u}{\hat{u}}\right)^{4}\left(\Delta_{g} w+\left(\frac{u}{\hat{u}}\right)^{2} g\left(\nabla(\hat{u} / u)^{2}, \nabla w\right)\right)
$$

and $\hat{u} / u=1$ outside $\Gamma\left(e^{-T+2}\right)$, while on $\Gamma\left(e^{-T+2}\right)$ :

$$
|\nabla w|_{g} \leqslant \frac{C}{m^{2}} T e^{-T}, \quad\left|\nabla(\hat{u} / u)^{2}\right|_{g} \leqslant C e^{-T} .
$$

It follows that (i) and (ii) also hold with $\hat{g}$ once we replace $c$ by say $c / 2$, provided $T$ is large enough.

We now turn to proving (i) and (ii) with respect to $g$. Let $g_{1}=u_{1}^{4} \delta$ be the one-black-hole Majumdar-Papapetrou metric, i.e., $u_{1}^{2}=1+m / r_{1}$. One easily calculates:

$$
\Delta_{g_{1}} \psi_{1}=-\frac{e^{-T}}{\left(r_{1}+m\right)^{3}}
$$

Let $\theta=\nabla r_{1} \cdot \nabla r_{2}$ denote the inner product of $\nabla r_{1}$ and $\nabla r_{2}$ with respect to $\delta$, then:

$$
\begin{aligned}
\Delta_{g} \psi_{1} & =u^{-6} \operatorname{div}_{\delta}\left(u^{2} \nabla \psi_{1}\right) \\
& =u^{-6} \operatorname{div}_{\delta}\left(\left(u_{1}^{2}+\frac{m}{r_{2}}\right) \nabla \psi_{1}\right) \\
& =\left(\frac{u_{1}}{u}\right)^{6} \Delta_{g_{1}} \psi_{1}+\frac{m}{u^{6}} \operatorname{div}_{\delta}\left(\frac{1}{r_{2} u_{1}^{2}} u_{1}^{2} \nabla \psi_{1}\right) \\
& =-\left(\frac{u_{1}}{u}\right)^{6}\left(1+\frac{m}{r_{2} u_{1}^{2}}\right) \frac{e^{-T}}{\left(r_{1}+m\right)^{3}}+\frac{m u_{1}^{2}}{u^{6}} \nabla\left(\frac{1}{r_{2} u_{1}^{2}}\right) \cdot \nabla \psi_{1} \\
& =-\left(\frac{u_{1}}{u}\right)^{6}\left(1+\frac{m}{r_{2} u_{1}^{2}}+\frac{m^{2} \log r_{1}}{r_{2}\left(r_{1}+m\right) u_{1}^{2}}-\frac{m r_{1}^{2} \log r_{1}}{r_{2}^{2}\left(r_{1}+m\right)} \theta\right) \frac{e^{-T}}{\left(r_{1}+m\right)^{3}}
\end{aligned}
$$


Note that $r_{1}>1$ on $B_{2}(1)$ hence, there, we can estimate $\Delta_{g} \psi_{1}$ above by the only positive term on the right-hand side:

$$
\Delta_{g} \psi_{1} \leqslant\left|\frac{m e^{-T} r_{2} \log r_{1}}{\left(r_{2}+m r_{2} / r_{1}+m\right)^{3} r_{1}\left(r_{1}+m\right)} \theta\right| \leqslant \frac{e^{-T}\left|\log r_{1}\right|}{m^{2} r_{1}^{2}} r_{2} .
$$

Furthermore, as $r_{1} \rightarrow 0$, then $\Delta_{g} \psi_{1} \rightarrow-e^{-T} / m^{3}$. It follows that, provided $m<1$, one can choose $\varepsilon>0$ independent of $T$ and $m$ such that:

$$
\Delta_{g} \psi_{1} \leqslant \begin{cases}e^{-T} / 4 m^{3}, & \text { when } r_{2}<\varepsilon \\ -e^{-T} / 2 m^{3}, & \text { when } r_{1}<\varepsilon .\end{cases}
$$

Now, we can choose $m>0$ small enough, so that $\Delta_{\hat{g}} \psi_{1} \leqslant 0$ when $r_{1}, r_{2} \geqslant \varepsilon$. By symmetry, we have analogous estimates for $\Delta_{g} \psi_{2}$. We conclude that:

$$
\begin{gathered}
\Delta_{g} w=\Delta_{g} \psi_{1}+\Delta_{g} \psi_{2} \leqslant 0, \quad \text { when } r_{1}, r_{2} \geqslant \varepsilon . \\
\Delta_{g} w \leqslant-\frac{e^{-T}}{4 m^{3}}, \quad \text { on } \Gamma(\varepsilon) .
\end{gathered}
$$

Properties (ii) and (iii) now follow provided $T>-\log \varepsilon+2$. This completes the proof of Proposition 2

Defining $\hat{E}^{\prime}=\hat{E}-\nabla \varphi$, we now have $\operatorname{div}_{\hat{g}}\left(\hat{E}^{\prime}\right)=0$, and in view of (15) and (13):

$$
\left\|R_{\hat{g}}-2\left|\hat{E}^{\prime}\right|_{\hat{g}}^{2}\right\|_{C_{-3}^{0, \alpha}} \leqslant C T^{2} e^{-T} .
$$

3.3. The Gauss Constraint. In this section, we prove that for each $T$ large enough, there is a positive solution $\phi \in 1+C_{-1}^{2, \alpha}(M)$ of Equation (7). We first prove the following estimate which gives a uniform bound on the inverse of the linearized operator associated with (7). The proof is adapted from [7, Proposition 8].

Proposition 3. Let

$$
h=\frac{1}{8} R_{\hat{g}}+\frac{3}{4}\left|\hat{E}^{\prime}\right|_{\hat{g}}^{2}
$$

Then there is a constant $C$ independent of $T$ such that if $\phi \in C_{-2 / 3}^{2, \alpha}$, then

$$
\|\phi\|_{C_{-2 / 3}^{2, \alpha}} \leqslant C\left\|\left(\Delta_{\hat{g}}-h\right) \phi\right\|_{C_{-8 / 3}^{0, \alpha}} .
$$

Remark 3. For $T$ bounded, this follows from Theorem 2 .

Proof. Suppose the contrary. Then there is a sequence $T_{j} \rightarrow \infty$, and $\phi_{j} \in$ $C_{-2 / 3}^{2, \alpha}$ satisfying

$$
\left\|\phi_{j}\right\|_{C_{-2 / 3}^{2, \alpha}}=1 \quad \forall j, \quad\left\|\left(\Delta_{\hat{g}}-h\right) \phi_{j}\right\|_{C_{-8 / 3}^{0, \alpha}} \rightarrow 0 \text { as } j \rightarrow \infty .
$$

By Proposition 1, we have

$$
\left\|\phi_{j}\right\|_{C_{-2 / 3}^{2, \alpha}} \leqslant C\left(\left\|\phi_{j}\right\|_{C_{-2 / 3}^{0}}+\left\|\left(\Delta_{\hat{g}}-h\right) \phi_{j}\right\|_{C_{-8 / 3}^{0, \alpha}}\right)
$$


with $C$ independent of $j$. Hence, in view of (19), we have $\varepsilon>0$ such that

$$
\varepsilon \leqslant\left\|\phi_{j}\right\|_{C_{-2 / 3}^{0}} \leqslant 1
$$

for all $j$. We now consider the following two cases:

(i) There is $\tau>0$ such that for any $\delta>0$, we have:

$$
\underset{j}{\limsup }\left\|\phi_{j}\right\|_{C^{0}(\hat{\Gamma}(\delta))} \geqslant \tau
$$

(ii) For every $\tau>0$, there exists $\delta>0$ so that:

$$
\underset{j}{\limsup }\left\|\phi_{j}\right\|_{C^{0}(\hat{\Gamma}(\delta))}<\tau .
$$

Note that $\hat{\Gamma}(\delta)$ is the union of the two necks cut at $r_{i}^{ \pm}=\delta$.

Case (i). For each integer $k$ large enough, take $\delta_{k}=e^{-k}$ in (i). Then there is $j_{k}$ large enough so that $T_{j_{k}}>k$ and so that there exists $p_{k} \in \hat{\Gamma}\left(\delta_{k}\right)$ with $\left|\phi\left(p_{j_{k}}\right)\right| \geqslant \tau / 2$. Without loss of generality we may assume that $p_{j_{k}} \in \Gamma_{1}^{+}\left(\delta_{k}\right)$. Furthermore, by passing to a subsequence, we may assume that $j_{k}=k$, i.e., $T_{k}>k$, and $p_{k} \in \Gamma_{1}^{+}\left(\delta_{k}\right)$. We define a coordinate $s$ on $\hat{M}$ by:

$$
s= \pm\left(\log r_{1}+T\right) \text { on } M_{ \pm} .
$$

Denote $s_{k}=s\left(p_{k}\right)$, then it follows that $0 \leqslant s_{k}<T_{k}-k$. Now let

$$
\Lambda_{k}=\left\{s_{k}-k / 2<s<s_{k}+k / 2\right\} .
$$

The part $\left\{s=s_{k}+k / 2\right\}$ of the boundary of $\Lambda_{k}$ has $r_{1}^{+}$coordinate equal to:

$$
\exp \left(s_{k}+k / 2-T_{k}\right)<e^{-k / 2} \rightarrow 0, \quad \text { as } k \rightarrow \infty .
$$

A similar estimate holds for the other part of the boundary. It follows that the metric $\hat{g}$ on $\Lambda_{k}$ converges to $m^{2}\left(d s^{2}+d \omega^{2}\right)$ as $k \rightarrow \infty$. Let $\left(\Lambda, g_{0}\right)$ denote the standard round cylinder with the metric $g_{0}=m^{2}\left(d s^{2}+d \omega^{2}\right)$. We will identify the points on $\Lambda_{k}$ with those of $\Lambda$ via the identity map induced by the $(s, \omega)$ coordinates. Observe that $\cup \Lambda_{k}=\Lambda$. Using the compactness of the embedding $C^{2, \alpha^{\prime}}\left(\Lambda_{k}\right) \hookrightarrow C^{2, \alpha}\left(\Lambda_{k}\right), 0 \leqslant \alpha^{\prime}<\alpha$, we can now select a subsequence, which we now denote $\phi_{k}$ again, and a function $\phi_{0}$ on $\Lambda$ such that $\phi_{k} \rightarrow \phi_{0}$ in $C^{2, \alpha^{\prime}}\left(\Lambda_{k}\right)$ for each fixed $k$. Furthermore, there is a point $p_{0}$ in the cross-section $\{s=0\}$ of $\Lambda$ such that $\left|\phi_{0}\left(p_{0}\right)\right| \geqslant \tau / 2$, hence $\phi_{0}$ is not identically zero. The scalar curvature $R_{\hat{g}}$ on $\Lambda_{k}$ converges to $2 / m^{2}$ and $\left|\hat{E}^{\prime}\right|_{\hat{g}}^{2}$ converges to $1 / m^{2}$. Thus, the coefficients of $\mathcal{T}=\Delta_{\hat{g}}-h$ converge uniformly on compact sets to the coefficients of

$$
\mathcal{T}_{0}=\Delta_{g_{0}}-\frac{1}{m^{2}}
$$

Hence we get $\mathcal{T} \phi_{k} \rightarrow \mathcal{T}_{0} \phi_{0}$ in $C^{0}\left(\Lambda_{k}\right)$. Since we also have $\mathcal{T} \phi_{k} \rightarrow 0$ in $C^{0, \alpha}\left(\Lambda_{k}\right)$ by (19), we conclude that $\phi_{0}$ satisfies the linear equation

$$
\Delta_{g_{0}} \phi_{0}-\frac{1}{m^{2}} \phi_{0}=0
$$


on $\Lambda$. Since $\phi_{0}$ is nontrivial, it has exponential growth in $s$ either as $s \rightarrow \infty$ or as $s \rightarrow-\infty$, in contradiction to (20).

Case (ii). We begin the treatment of this case with the following lemma:

Lemma 4. Suppose $\phi_{j}$ satisfies (19) and (ii), and let $A_{\delta}^{+} \subset M_{+}$be the twice perforated ball $A_{\delta}=B_{0}(3) \backslash D(\delta / 2)$. Then for each $\delta>0$, there holds $\left\|\phi_{j}\right\|_{C^{1, \alpha}\left(A_{\delta}\right)} \rightarrow 0$ as $j \rightarrow \infty$.

Proof. Suppose not, and let $j_{k}$ be a subsequence such that $\phi_{j_{k}}$ converges to $\phi_{0}$ in $C^{1, \alpha^{\prime}}\left(A_{\delta}\right), \alpha^{\prime}<\alpha$. Then $\phi_{0}$ is not identically zero on $A_{\delta}$, hence since $h>0$ on $A_{\delta}$, we have:

$$
\lim _{k} \int_{A_{\delta}} h \phi_{j_{k}}^{2}=\int_{A_{\delta}} h \phi_{0}^{2}>0
$$

We now proceed to show that

$$
\limsup \int_{A_{\delta}} h \phi_{j}^{2}=0
$$

leading to a contradiction. Without loss of generality, we may assume, by passing to a subsequence, that:

$$
\int_{A_{\delta}} h \phi_{j}^{2} \rightarrow \limsup _{j} \int_{A_{\delta}} h \phi_{j}^{2}
$$

If $\chi$ is any smooth cut-off function of compact support in $M_{+}$, with $0 \leqslant \chi \leqslant$ 1 , and $\chi=1$ in $A_{\delta}$, then:

$$
\begin{aligned}
\int_{A_{\delta}} h \phi_{j}^{2} & \leqslant \int_{M_{+}} \chi^{2}\left(\left|\nabla \phi_{j}\right|_{\hat{g}}^{2}+h \phi_{j}^{2}\right) \\
& =-\int_{M_{+}} \chi^{2} \phi_{j}\left(\Delta_{\hat{g}}-h\right) \phi_{j}-2 \int_{M_{+}} \chi \phi_{j} \hat{g}\left(\nabla \chi, \nabla \phi_{j}\right) .
\end{aligned}
$$

We will now choose cut-offs $\chi_{k}$, and a subsequence $j_{k}$ along which both of the terms on the right-hand side tend to zero, proving (21). By (ii), for each integer $k$ we can choose $0<\delta_{k}<\delta$ such that $\left|\phi_{j}\right|<1 / k$ on $\hat{\Gamma}\left(\delta_{k}\right)$ for all $j$ large enough. Now, we can choose $\chi_{k}$ supported on $B_{0}(k) \backslash D\left(\delta_{k} / 2\right)$, with:

$$
\operatorname{supp} \nabla \chi_{k} \subset\left[B_{0}(k) \backslash B_{0}(3)\right] \cup\left[D\left(\delta_{k}\right) \backslash D\left(\delta_{k} / 2\right)\right],
$$

and satisfying:

$$
\begin{aligned}
& \left|\nabla \chi_{k}\right|_{\hat{g}} \leqslant C / k, \quad \text { on } B_{0}(k) \backslash B_{0}(3), \\
& \left|\nabla \chi_{k}\right|_{\hat{g}} \leqslant C, \quad \text { on } D\left(\delta_{k}\right) \backslash D\left(\delta_{k} / 2\right) .
\end{aligned}
$$

Finally, by (19), we can choose $j_{k}>j_{k-1}$, so that

$$
\left(\int_{\mathbb{R}^{3} \backslash B_{0}(3)} \sigma^{-10 / 3}\right)\left\|\left(\Delta_{\hat{g}}-h\right) \phi_{j_{k}}\right\|_{C_{-8 / 3}^{0, \alpha}} \leqslant \frac{1}{k} .
$$


It then follows that:

$$
-\int_{M_{+}} \chi_{k}^{2} \phi_{j_{k}}\left(\Delta_{\hat{g}}-h\right) \phi_{j_{k}}<\frac{1}{k}
$$

and:

$$
\begin{aligned}
-\int_{M_{+}} \chi_{k} \phi_{j_{k}} \hat{g}\left(\nabla \chi_{k}, \nabla \phi_{j_{k}}\right) & \leqslant C \int_{D\left(\delta_{k}\right) \backslash D\left(\delta_{k} / 2\right)}\left|\phi_{j_{k}}\right|+C k^{-1} \int_{B_{0}(k) \backslash B_{0}(3)} \sigma^{-7 / 3} \\
& \leqslant C k^{-1}+C k^{-1} \int_{3}^{k} \frac{d r}{r^{1 / 3}} \\
& \leqslant C\left(k^{-1}+k^{-1 / 3}\right) .
\end{aligned}
$$

This completes the proof of Lemma 4

Now choose $\delta>0$ so that

$$
\limsup _{j}\left\|\phi_{j}\right\|_{C^{0}(\hat{\Gamma}(\delta))}<\varepsilon
$$

where $\varepsilon$ is defined by (20), and define a new manifold $\left(M_{*}, g_{*}\right)$ diffeomorphic to $\mathbb{R}^{3}$ by extending smoothly the metric $\hat{g}$ on $\mathbb{R}^{3} \backslash D(\delta / 2)$ over $D(\delta / 2)$. Then extend smoothly to $D(\delta / 2)$ also the potential function $h$ so that the extended potential $h_{*}$ satisfies $h_{*} \geqslant 0$ on $\mathbb{R}^{3}$. Let $\chi$ be a smooth cut-off function on $\mathbb{R}^{3}$ with $0 \leqslant \chi \leqslant 1, \chi=1$ outside $D(\delta)$, and $\chi=0$ on $D(\delta / 2)$. Taking the values of $\phi_{j}$ from $M_{+}$, we can view $\chi \phi_{j}$ as a function on $M_{*}$, and we find:

$$
\left(\Delta_{g_{*}}-h_{*}\right) \chi \phi_{j}=\left(\Delta_{\hat{g}}-h\right) \chi \phi_{j}=\chi(\Delta-h) \phi_{j}+2 \hat{g}\left(\nabla \chi, \nabla \phi_{j}\right)+\phi_{j} \Delta_{\hat{g}} \chi .
$$

Hence, we can estimate:

$$
\left\|\left(\Delta_{g_{*}}-h_{*}\right) \chi \phi_{j}\right\|_{C_{-8 / 3}^{0, \alpha}\left(M_{*}\right)} \leqslant\left\|(\Delta-h) \phi_{j}\right\|_{C_{-8 / 3}^{0, \alpha}}+C\left\|\phi_{j}\right\|_{C^{1, \alpha}\left(A_{\delta}\right)} \rightarrow 0,
$$

by (19) and Lemma 4. It then follows by Theorem 2 part (b) applied to $\left(M_{*}, g_{*}\right)$ that:

$$
\left\|\chi \phi_{j}\right\|_{C_{-2 / 3}^{2, \alpha}\left(M_{*}\right)} \rightarrow 0
$$

Thus, we obtain:

$$
\left\|\phi_{j}\right\|_{C_{-2 / 3}^{0}\left(M_{+} \backslash \Gamma^{+}(\delta)\right)} \rightarrow 0
$$

Similarly, we obtain:

$$
\left\|\phi_{j}\right\|_{C_{-2 / 3}^{0}\left(M_{-} \backslash \Gamma^{-}(\delta)\right)} \rightarrow 0
$$

and it follows that:

$$
\left\|\phi_{j}\right\|_{C_{-2 / 3}^{0}(\hat{M} \backslash \hat{\Gamma}(\delta))} \rightarrow 0 .
$$

Combining with (22), we conclude that:

$$
\underset{j}{\limsup }\left\|\phi_{j}\right\|_{C_{-2 / 3}^{0}(\hat{M})}<\varepsilon
$$

in contradiction to (20). This completes the proof of Proposition 3

We can now prove the main result of this section. 
Proposition 5. For each $m>0$ small enough and for each $T$ large enough there is a solution $\phi \in 1+C_{-1}^{2, \alpha}(M)$ of:

$$
L_{\hat{g}} \phi=-\frac{\left|\hat{E}^{\prime}\right|_{\hat{g}}^{2}}{4 \phi^{3}}
$$

Furthermore, as $T \rightarrow \infty$, this solution satisfies $\|\phi-1\|_{C_{-1}^{2, \alpha}} \rightarrow 0$.

Proof. Let $\mathcal{N}: 1+C_{-2 / 3}^{2, \alpha} \rightarrow C_{-8 / 3}^{0, \alpha}$ be the following nonlinear operator:

$$
\mathcal{N}(1+\psi)=L_{\hat{g}}(1+\psi)+\frac{\left|\hat{E}^{\prime}\right|_{\hat{g}}^{2}}{4(1+\psi)^{3}} .
$$

The linearization of $\mathcal{N}$ about 1 is:

$$
d \mathcal{N}=L_{\hat{g}}-\frac{3}{4}\left|\hat{E}^{\prime}\right|_{\hat{g}}^{2}: C_{-2 / 3}^{2, \alpha} \rightarrow C_{-8 / 3}^{0, \alpha}
$$

and according to Proposition $3, d \mathcal{N}^{-1}: C_{-8 / 3}^{0, \alpha} \rightarrow C_{-2 / 3}^{2, \alpha}$ is uniformly bounded, i.e., there is a constant $C$ independent of $T$ such that

$$
\left\|d \mathcal{N}^{-1} \psi\right\|_{C_{-2 / 3}^{2, \alpha}} \leqslant C\|\psi\|_{C_{-8 / 3}^{0, \alpha}}
$$

Now consider the 'quadratic part' of $\mathcal{N}$ :

$$
\mathcal{Q}(\psi)=\mathcal{N}(1+\psi)-\mathcal{N}(1)-d \mathcal{N}(\psi)
$$

We have:

$$
\mathcal{Q}(\psi)=\frac{\left|\hat{E}^{\prime}\right|_{\hat{g}}^{2}\left(6+8 \psi+3 \psi^{2}\right)}{4(1+\psi)^{3}} \psi^{2},
$$

hence it follows that there is a constant $C$ independent of $T$ such that if $\eta>0$ is sufficiently small, and $\|\psi\|_{C_{-2 / 3}^{2, \alpha}}<\eta$, then the following holds:

$$
\begin{gathered}
\|\mathcal{Q}(\psi)\|_{C_{-8 / 3}^{0, \alpha}} \leqslant C \eta^{2} \\
\left\|\mathcal{Q}\left(\psi_{1}\right)-\mathcal{Q}\left(\psi_{2}\right)\right\|_{C_{-8 / 3}^{0, \alpha}} \leqslant 2 C \eta\left\|\psi_{1}-\psi_{2}\right\|_{C_{-2 / 3}^{2, \alpha}} .
\end{gathered}
$$

Now, choose $0<\lambda<1, \eta>0$ such that $\eta<\lambda / 2 C^{2}$, and $T>0$ such that $T^{2} e^{-T}<\eta^{2}$. Then, if $\mathcal{B}$ is the ball of radius $\eta$ in $C_{-2 / 3}^{2, \alpha}$, the map $\mathcal{F}$ given by:

$$
\mathcal{F}(\psi)=-d \mathcal{N}^{-1}(\mathcal{N}(1)+\mathcal{Q}(\psi))
$$

maps $\mathcal{B}$ into $\mathcal{B}$ and is a contraction. Indeed, in view of (18) and (25), we have:

$$
\|\mathcal{F}(\psi)\|_{C_{-2 / 3}^{2, \alpha}} \leqslant C\left(\|\mathcal{N}(1)\|_{C_{-8 / 3}^{0, \alpha}}+\|\mathcal{Q}(\psi)\|_{C_{-8 / 3}^{0, \alpha}}\right) \leqslant C^{2}\left(T^{2} e^{-T}+\eta^{2}\right)<\eta,
$$

and in view of (26)

$$
\begin{aligned}
\left\|\mathcal{F}\left(\psi_{1}\right)-\mathcal{F}\left(\psi_{2}\right)\right\|_{C_{-2 / 3}^{2, \alpha}} \leqslant C & \left\|\mathcal{Q}\left(\psi_{1}\right)-\mathcal{Q}\left(\psi_{2}\right)\right\|_{C_{-8 / 3}^{0, \alpha}} \\
& \leqslant 2 C^{2} \eta\left\|\psi_{1}-\psi_{2}\right\|_{C_{-2 / 3}^{2, \alpha}}<\lambda\left\|\psi_{1}-\psi_{2}\right\|_{C_{-2 / 3}^{2, \alpha}} .
\end{aligned}
$$


It follows that $\mathcal{F}$ has a fixed point $\psi$ in $\mathcal{B}$ which satisfies

$$
\mathcal{N}(1+\psi)=\mathcal{N}(1)+d \mathcal{N}(\psi)+\mathcal{Q}(\psi)=0 .
$$

Furthermore, note that if $T \rightarrow \infty$, one can choose $\eta \rightarrow 0$. Thus we have $\|\psi\|_{C_{-2 / 3}^{2, \alpha}} \rightarrow 0$. It also follows from (24) that:

$$
\Delta_{\hat{g}} \psi=\frac{1}{8} R_{\hat{g}} \psi+\frac{\left|\hat{E}^{\prime}\right|_{\hat{g}}^{2}}{4(1+\psi)^{3}}-\frac{1}{8} R_{\hat{g}} .
$$

We will now show that the right-hand side above tends to zero as $T \rightarrow \infty$ in $C_{-3}^{0, \alpha} \cap L^{1}$. Indeed, we have:

$$
\begin{gathered}
\frac{1}{8} R_{\hat{g}} \psi+\frac{\left|\hat{E}^{\prime}\right|_{\hat{g}}^{2}}{4(1+\psi)^{3}}-\frac{1}{8} R_{\hat{g}} \\
=\frac{1}{8} R_{\hat{g}} \psi-\frac{1}{8}\left[R_{\hat{g}}-2\left|\hat{E}^{\prime}\right|_{\hat{g}}^{2}\right]-\frac{\left|\hat{E}^{\prime}\right|_{\hat{g}}^{2}}{4\left(1+\psi^{3}\right)}\left((1+\psi)^{3}-1\right) \\
=\frac{1}{8} R_{\hat{g}} \psi-\frac{1}{8}\left[R_{\hat{g}}-2|\hat{E}|_{\hat{g}}^{2}\right]-\frac{1}{4} \hat{g}(2 \hat{E}+\nabla \varphi, \nabla \varphi)+\frac{\left|\hat{E}^{\prime}\right|_{\hat{g}}^{2}\left(3+3 \psi+\psi^{2}\right)}{4\left(1+\psi^{3}\right)} \psi
\end{gathered}
$$

We now proceed to check that each of the terms above tends to zero in $C_{-3}^{0, \alpha} \cap L^{1}$ as $T \rightarrow \infty$. The second term above tends to zero by (13), and the fact that it is supported on a set of uniformly bounded volume. For the other three terms, we use the fact, that if $f_{i} \in C_{-\beta_{i}}^{0, \alpha}, i=1,2$, and $\beta_{1}+\beta_{2}>3$, then

$$
\left\|f_{1} f_{2}\right\|_{C_{-3}^{0, \alpha} \cap L^{1}}=\left\|f_{1} f_{2}\right\|_{C_{-3}^{0, \alpha}}+\left\|f_{1} f_{2}\right\|_{L^{1}} \leqslant C\left\|f_{1}\right\|_{C_{-\beta_{1}}^{0, \alpha}}\left\|f_{2}\right\|_{C_{-\beta_{2}}^{0, \alpha}} .
$$

If one of the factors on the right-hand side of the inequality tends to zero and the other is bounded, then the left-hand side of the inequality tends to zero. The first and last term above are of the form $f \psi$, with $\|f\|_{C_{-4}^{0, \alpha}}$ bounded and $\|\psi\|_{C_{-2 / 3}^{0, \alpha}} \rightarrow 0$. The third term is of the form $f|\nabla \varphi|_{\hat{g}}$ with $\|f\|_{C_{-2}^{0, \alpha}}$ bounded and $\|\nabla \varphi\|_{C_{-2}^{0, \alpha}} \rightarrow 0$. We conclude that:

$$
\left\|\Delta_{\hat{g}} \psi\right\|_{C_{-3}^{0, \alpha} \cap L^{1}} \rightarrow 0
$$

The result will now follow from the following Lemma.

Lemma 6. Suppose that $\psi \in C_{-2 / 3}^{0, \alpha}$ and $\Delta_{\hat{g}} \psi \in C_{-3}^{0, \alpha} \cap L^{1}$. Then there is a constant $C$ independent of $T$ such that:

$$
\|\psi\|_{C_{-1}^{2, \alpha}} \leqslant C\left(\left\|\Delta_{\hat{g}} \psi\right\|_{C_{-3}^{0, \alpha} \cap L^{1}}+\|\psi\|_{C_{-2 / 3}^{2, \alpha}}\right) .
$$

Proof of Lemma [6. The proof of this lemma is based on the proof of Proposition 29 in [13, Appendix]. There, it is proved that if $v$ is a function on $\mathbb{R}^{3}$ with $v \in C_{-2 / 3}^{0, \alpha}$, and $\Delta_{\hat{g}} v \in C_{-3}^{0, \alpha} \cap L^{1}$, then:

$$
\|v\|_{C_{-1}^{2, \alpha}} \leqslant C\left\|\Delta_{\hat{g}} v\right\|_{C_{-3}^{0, \alpha} \cap L^{1}}
$$


Let $\Omega_{R}=\{r>R\} \subset M_{+}$. Clearly, for any finite $R \geqslant 3$, the two norms $\|\cdot\|_{C_{-1}^{2, \alpha}\left(M_{+} \backslash \Omega_{R}\right)}$ and $\|\cdot\|_{C_{-2 / 3}^{2, \alpha}\left(M_{+} \backslash \Omega_{R}\right)}$ are equivalent. Let $\chi$ be a smooth cutoff function with $0 \leqslant \chi \leqslant 1, \chi=0$ on $M \backslash \Omega_{3}$ and $\chi=1$ on $\Omega_{4}$. Then $v=\chi \psi$ can be viewed as a function on $\mathbb{R}^{3}$, and $v \in C_{-2 / 3}^{2, \alpha}$. We have:

$$
\Delta_{\hat{g}} v=\chi \Delta_{\hat{g}} \psi+2 \hat{g}(\nabla \chi, \nabla \psi)+\psi \Delta_{\hat{g}} \chi
$$

The last two terms above are supported on the annulus $\Omega_{3} \backslash \Omega_{4}$, hence we can estimate:

$$
\left\|2 \hat{g}(\nabla \chi, \nabla \psi)+\psi \Delta_{\hat{g}} \chi\right\|_{C_{-3}^{0, \alpha} \cap L^{1}} \leqslant C\|\psi\|_{C_{-2 / 3}^{2, \alpha}}
$$

while for the first term we clearly have:

$$
\left\|\chi \Delta_{\hat{g}} \psi\right\|_{C_{-3}^{0, \alpha} \cap L^{1}} \leqslant C\left\|\Delta_{\hat{g}} \psi\right\|_{C_{-3}^{0, \alpha} \cap L^{1}} .
$$

Thus, we obtain:

$$
\left\|\Delta_{\hat{g}} v\right\|_{C_{-3}^{0, \alpha} \cap L^{1}} \leqslant C\left(\left\|\Delta_{\hat{g}} \psi\right\|_{C_{-3}^{0, \alpha} \cap L^{1}}+\|\psi\|_{C_{-2 / 3}^{2, \alpha}}\right) .
$$

We now conclude from (28) that

$$
\|\psi\|_{C_{-1}^{2, \alpha}\left(\Omega_{4}\right)} \leqslant C\left\|\Delta_{\hat{g}} v\right\|_{C_{-3}^{0, \alpha} \cap L^{1}} \leqslant C\left(\left\|\Delta_{\hat{g}} \psi\right\|_{C_{-3}^{0, \alpha} \cap L^{1}}+\|\psi\|_{C_{-2 / 3}^{2, \alpha}}\right) .
$$

Hence, we have:

$$
\begin{aligned}
\|\psi\|_{C_{-1}^{2, \alpha}\left(M_{+}\right)} & \leqslant C\left(\|\psi\|_{C_{-1}^{2, \alpha}\left(\Omega_{4}\right)}+\|\psi\|_{C_{-1}^{2, \alpha}\left(M_{+} \backslash \Omega_{5}\right)}\right) \\
& \leqslant C\left(\left\|\Delta_{\hat{g}} \psi\right\|_{C_{-3}^{0, \alpha} \cap L^{1}}+\|\psi\|_{C_{-2 / 3}^{2, \alpha}}\right)
\end{aligned}
$$

A similar estimate holds on $M_{-}$. This completes the proof of Lemma 6 .

Taking $\phi=1+\psi$, we see that $\phi$ satisfies Equation (23), and by (27) and Lemma 6] we have $\|\phi-1\|_{C_{-1}^{2, \alpha}} \rightarrow 0$ as $T \rightarrow \infty$. This completes the proof of Proposition 5 .

We have shown that for each $m>0$ sufficiently small and for each $T$ sufficiently large, there is a two-ended solution $(\widetilde{M}, \tilde{g}, \widetilde{E}, 0)$ of the EinsteinMaxwell constraints, with $\widetilde{M}=\hat{M}, \tilde{g}=\phi^{4} \hat{u}^{4} \delta=\tilde{\phi}^{4} g$, where $\tilde{\phi}=\phi \hat{u} / u$ and $g$ is the Majumdar-Papapetrou solution. We note that for any $\eta>0$, we can assure that:

$$
\|\tilde{\phi}-1\|_{C_{-1}^{2, \alpha}}<\eta, \quad\|E-\widetilde{E}\|_{C_{-2}^{1, \alpha}}<\eta
$$

by taking $T$ large enough. For the sake of simplicity, we now rename $\tilde{\phi}$ to be $\phi$. Furthermore, we note that this solution admits an involutive, chargereversing, symmetry with fixed-point set $\Sigma_{0}$. 


\section{The Outermost Horizon}

In this section, we show that with $m>0$ fixed and sufficiently small, we can adjust the perturbation parameter $\eta>0$ to be small enough so that the area $\tilde{A}$ of the outermost horizon in the conformal perturbation $\tilde{g}$ is no greater than $8 \pi \lambda^{2} m^{2}$ where $\lambda-1>0$ is arbitrarily small, i.e., $\widetilde{R} \leqslant \sqrt{2} \lambda m$. Furthermore, if the perturbation parameter $\eta>0$ is small enough we can assure that the total mass $\widetilde{m}$ of $\tilde{g}$ is no greater than $2 \lambda m$ and that the charge $\widetilde{Q}$ of $\widetilde{E}$ satisfies $\widetilde{Q} \geqslant Q / \lambda$, where $Q=2 m$. Now, the function $f_{Q}(x)=x+Q^{2} / \lambda^{2} x$ is non-increasing for $0<x<Q / \lambda$. Thus, if we choose $\lambda$ so that

$$
1<\lambda<(\sqrt{2}-1 / 2)^{-1 / 4}<2^{1 / 4},
$$

we get:

$$
\widetilde{m}-\frac{1}{2}\left(\widetilde{R}+\frac{\widetilde{Q}^{2}}{\widetilde{R}}\right) \leqslant \widetilde{m}-\frac{1}{2}\left(\widetilde{R}+\frac{Q^{2}}{\lambda^{2} \widetilde{R}}\right) \leqslant \lambda m\left(2-\frac{1}{\sqrt{2}}-\frac{\sqrt{2}}{\lambda^{4}}\right)<0,
$$

This proves Theorem 1.

We will use the following elementary lemma.

Lemma 7. Let $M$ be a Riemannian manifold, and let $S \subset M$ be a compact hypersurface with unit normal $n$. Let $v$ be a smooth function on $M$, and suppose that the maximum of $v$ over $S$ is taken at a point $q \notin \partial S$ where $\nabla v \neq 0$. Then

$$
|H(q)| \geqslant\left.\frac{\Delta v-\nabla_{n}^{2} v}{|\nabla v|}\right|_{q}
$$

where $H$ is the mean curvature of $S$.

Proof. Let $\not \Delta$ denote the Laplacian with respect to the metric induced on $S$. We have at $q$ :

$$
\Delta v=\nabla_{n}^{2} v+H \nabla_{n} v+\not \Delta v \leqslant \nabla_{n}^{2} v+H \nabla_{n} v,
$$

since $\not \Delta v \leqslant 0$ there, and since, without loss of generality, we may take $n=\nabla v /|\nabla v|$. Thus, we obtain:

$$
\Delta v \leqslant \nabla_{n}^{2} v+H|\nabla v|,
$$

and (30) follows,

The right hand side of (30) is easily recognized as the mean curvature of the level set of $v$ at $q$. Thus this lemma is simply another version of the familiar geometric fact that when two surfaces are tangent at $q$ and one lies entirely on one side from the other, then their mean curvature at $q$ are correspondingly ordered. We prefer the statement in the lemma since it simplifies some of the explicit computations below. A first application is the following lemma. 
Lemma 8. Let $p_{0}=(0,0,0), p_{1}=(0,0,1), p_{2}=(0,0,-1) \in \mathbb{R}^{3}$, and let $0<\varepsilon<1 / 3$. Then, for any compact surface $S \subset B_{0}(3) \backslash D(\varepsilon)$ such that $\partial S \subset \partial D(\varepsilon)$, there holds:

$$
\sup _{S}|H| \geqslant \frac{1}{6}
$$

where $H$ is the mean curvature of $S$.

Proof. We consider two cases: (i) $\max _{S} v>0$; and (ii) $v \leqslant 0$ on $S$. For case (i), take $v=x^{2}+y^{2}-z^{2} / 2$. Let $q \in S$ be such that $v(q)=\max _{S} v$. Since $v<0$ on $\partial D(\varepsilon)$ we conclude that $q \notin \partial S$. Since $\Delta v=3, \nabla_{n}^{2} v \leqslant 2$, and $|\nabla v| \leqslant 2 r<6$, Lemma 7 now yields:

$$
H(q) \geqslant \frac{1}{6} \text {. }
$$

Now in case (ii) note that since $S$ is smooth, it is contained in the double cone $v<0$. Without loss of generality $S_{1}=S \cap\{z>0\} \neq \emptyset$, and we now take $v=r_{1}^{2}=x^{2}+y^{2}+(z-1)^{2}$, and let $q$ as above be such that $v(q)=\max _{S} v$. If $q \in \partial S_{1} \subset \partial B_{1}(\varepsilon)$, then $S_{1} \subset \partial B_{1}(\varepsilon)$ and $H=2 / \varepsilon \geqslant 6$ at every interior point of $S$. On the other hand, if $q \notin \partial S_{1}$, then since $\Delta w=6$, $\nabla_{n}^{2} w=2$, and $|\nabla w|=2 r_{1}$, we obtain from Lemma 7 that:

$$
H(q) \geqslant \frac{2}{r_{1}}>\frac{1}{2} \text {. }
$$

Proposition 9. If $m$ is sufficiently small, then for each $\varepsilon>0$ there is $\eta>0$ such that if $\|\phi-1\|_{C_{-1}^{2, \alpha}}<\eta$, then any closed surface $S \subset M$ which is minimal in the conformal perturbation $\left(M, \phi^{4} g, \phi^{-6} E\right)$ of $(M, g, E)$ is contained in $D(\varepsilon)$.

Proof. The proof is established in three stages. We first show that $S$ cannot enter the region outside $B_{0}(3)$. We then do the same for for the twiceperforated ball $B_{0}(3) \backslash D(1 / 4)$. Finally, we prove the result in each of the two balls $B_{1}(1 / 4)$ and $B_{2}(1 / 4)$ separately. We will use the Euclidean metric $\delta$, the Majumdar-Papapetrou metric $g=u^{4} \delta$, and also its perturbation $\tilde{g}=\phi^{4} g$. In order to avoid confusion we will use the dot product to denote the inner product with respect to $\delta$, and indicate other metric objects by subscripts. We denote $\nu=\phi u$, and note that

$$
H_{\tilde{g}}=\operatorname{div}_{\tilde{g}}\left(n_{\tilde{g}}\right)=\frac{1}{\nu^{6}} \operatorname{div}_{\delta}\left(\nu^{4} n_{\delta}\right)=\frac{1}{\nu^{2}} H_{\delta}+\frac{4}{\nu} \tilde{g}\left(\nabla \nu, n_{\tilde{g}}\right),
$$

where $H_{\tilde{g}}$ and $H_{\delta}$ denote the mean curvatures of $S$ in the metrics $\tilde{g}$ and $\delta$ respectively.

Suppose first that $\max _{S} r \geqslant 3$ where $r$ is the Euclidean distance from $p_{0}$. Then, in view of $\Delta_{\delta} r=2 / r,|\nabla r|_{\delta}=1, \nabla^{2} r=0$, we have according to Lemma 7 that at the point $q$ with maximum $r$ :

$$
\left|H_{\delta}(q)\right| \geqslant \frac{2}{r}
$$


Now, $u^{2}=1+m / r_{1}+m / r_{2}$, hence outside $B_{0}(3)$, we have:

$$
|\nabla \log u|_{g}=\frac{1}{2} \frac{\left|\nabla u^{2}\right|_{\delta}}{u^{4}} \leqslant \frac{1}{2} \frac{m\left(1 / r_{1}^{2}+1 / r_{2}^{2}\right)}{\left(1+m / r_{1}+m / r_{2}\right)^{2}} \leqslant \frac{m}{(r-1)^{2}} \leqslant \frac{3 m}{4 r} .
$$

Thus, using (31) and $|\phi-1|, r^{2}|\nabla \phi|_{g} \leqslant\|\phi-1\|_{C_{-1}^{2, \alpha}}<\eta$, we can estimate:

$$
\begin{aligned}
\left|H_{\tilde{g}}(q)\right| & \geqslant \frac{\left|H_{\delta}\right|}{\nu^{2}}-4|\nabla \log \nu|_{\tilde{g}} \\
& \geqslant \frac{2}{r(1+2 m / 3)(1+\eta)^{2}}-\frac{4}{\phi^{2}}\left(|\nabla \log \phi|_{g}+|\nabla \log u|_{g}\right) \\
& \geqslant \frac{2}{r(1+2 m / 3)(1+\eta)^{2}}-\frac{4}{(1-\eta)^{3}}\left(\frac{\eta}{r^{2}}+\frac{3 m}{4 r}\right) \\
& \geqslant \frac{2}{r}\left(\frac{1}{(1+2 m / 3)(1+\eta)^{2}}-\frac{4 \eta+3 m}{2(1-\eta)^{3}}\right)
\end{aligned}
$$

Clearly if $m$ and $\eta$ are small enough, then $\left|H_{\tilde{g}}(q)\right|>0$, a contradiction. We conclude that $S \subset B_{0}(3)$.

Suppose now that $S$ enters $B_{0}(3) \backslash D(1 / 4)$. Then a similar estimate yields a point $q$ in that region where:

$$
\left|H_{\delta}(q)\right| \leqslant 4\left(1+\frac{2 m}{3}\right)\left(\frac{\eta}{9(1-\eta)}+16 m\right)
$$

Hence, if $\eta$ and $m$ are small enough, then we have $\left|H_{\delta}(q)\right|<1 / 6$ in contradiction to Lemma 8

Therefore, we can now fix $m$ and $\eta_{0}$ small enough such that if $\eta<\eta_{0}$ then $S$ must lie in $D(1 / 4)$. Consider the closed surface $S_{1}=S \cap B_{1}(1 / 4)$ with $H_{\tilde{g}}=0$. As above, we can estimate:

$$
\left|H_{g}\right| \leqslant 4|\nabla \log \phi|_{g}<\frac{4 \eta}{1-\eta}
$$

We will now apply Lemma 7 to the surface $S_{1}$ and the function $r_{1}$ in $B_{1}(1 / 4)$ equipped with the metric $g$. Let $q$ be the point where $r_{1}(q)=\max _{S_{1}} r_{1}<$ $1 / 4$. We compute at $q$, using $n_{g}=\nabla r_{1} /\left|\nabla r_{1}\right|_{g}$ :

$$
\begin{gathered}
\Delta_{g} r_{1}=\frac{1}{u^{6}} \operatorname{div}_{\delta}\left(u^{2} \nabla r_{1}\right)=\frac{1}{u^{6}}\left(\frac{2 u^{2}}{r_{1}}+\nabla u^{2} \cdot \nabla r_{1}\right) \\
\nabla_{n_{g}}^{2} r_{1}=\nabla_{n_{g}}\left|\nabla r_{1}\right|=\nabla_{n_{g}} u^{-2}=-\frac{g\left(\nabla u^{2}, \nabla r_{1}\right)}{u^{4}\left|\nabla r_{1}\right|}=-\frac{\nabla u^{2} \cdot \nabla r_{1}}{u^{6}} .
\end{gathered}
$$


Thus, using $m<2$, we can estimate:

$$
\begin{aligned}
\frac{\nabla r_{1}-\nabla_{n_{g}}^{2} r_{1}}{\left|\nabla r_{1}\right|} & =\frac{1}{u^{4}}\left(\frac{2 u^{2}}{r_{1}}+2 \nabla u^{2} \cdot \nabla r_{1}\right) \\
& =\frac{1}{u^{4}}\left(\frac{2 u^{2}}{r_{1}}-2 m\left(\frac{1}{r_{1}^{2}}+\frac{\nabla r_{1} \cdot \nabla r_{2}}{r_{2}^{2}}\right)\right) \\
& \geqslant \frac{1}{u^{4}}\left(\frac{2}{r_{1}}-2 m\right) \\
& \geqslant \frac{r_{1}}{(1+m)^{2}} .
\end{aligned}
$$

We now obtain from Lemma 7

$$
\frac{r_{1}(q)}{(1+m)^{2}} \leqslant\left|H_{g}(q)\right| \leqslant \frac{4 \eta}{1-\eta} .
$$

Therefore, with $m$ fixed, we see that $\max _{S_{1}} r_{1} \rightarrow 0$ as $\eta \rightarrow 0$. The same argument can be applied to $S_{2}=S \cap B_{2}(1 / 4)$. This proves Proposition 9

Proposition 10. Let $\lambda>1$. Then for $m$ and $\eta$ sufficiently small, the mass $\widetilde{m}$ of $\tilde{g}$, the area $\tilde{A}$ of the outermost horizon in $\tilde{g}$, and the charge $\widetilde{Q}$ satisfy:

$$
\widetilde{m} \leqslant 2 \lambda m, \quad \tilde{A} \leqslant 8 \pi \lambda^{2} m^{2}, \quad \widetilde{Q} \geqslant Q / \lambda .
$$

Proof. The metric of the perturbed space is $\tilde{g}=\phi^{4} u^{4} \delta$, therefore its mass $\widetilde{m}$ is:

$$
\widetilde{m}=2 m-\frac{1}{2 \pi} \lim _{r \rightarrow \infty} \int_{S_{r}} \frac{\partial \phi}{\partial r} d A_{0},
$$

where $A_{0}$ is the area element of $S_{r}$ in the flat metric $\delta$. Since $\partial \phi / \partial r$ can be estimated on $S_{r}$ by $r^{-2} u^{2}\|\phi\|_{C_{-1}^{2, \alpha}}<r^{-2} u^{2} \eta$, we find:

$$
\widetilde{m} \leqslant 2 m\left(1+\frac{\eta}{m}\right)
$$

Thus, $\widetilde{m} \leqslant 2 \lambda m$ provided $\eta \leqslant m(\lambda-1)$. Similarly $\widetilde{Q} \geqslant Q / \lambda$ follows from (29).

We now note that $\tilde{g}$ admits one horizon. Indeed, the surface $\Sigma_{0}=\{s=0\}$ cutting the neck at its midpoint is totally geodesic, since it is the fixed-point set of the isometry sending any point $p$ on one side of it to the corresponding point on the other. In particular, this surface is minimal and encloses the end $\infty_{-}$, hence it is a horizon.

Now let $S$ be the outermost horizon. Then $S$ is outer minimizing. According to Proposition 9, if $m>0$ and $\eta>0$ are sufficiently small, then $S \subset D(\varepsilon)$, where $\varepsilon \leqslant 4 \eta(1+m)^{2} /(1-\eta)$. Thus, $\partial D(\varepsilon)$ encloses $S$, and we 
conclude:

$$
\begin{gathered}
\tilde{A}=A_{\tilde{g}}(S) \leqslant A_{\tilde{g}}(\partial D(\varepsilon))=\int_{\partial D(\varepsilon)} \phi^{2} u^{4} d A_{0} \\
\leqslant(1+\eta)^{4} \int_{\partial D(\varepsilon)} u^{4} d A_{0} \leqslant 8 \pi m^{2}(1+\eta)^{4}\left(1+\varepsilon\left(1+\frac{1}{m}\right)\right)^{2} \\
\leqslant 8 \pi m^{2}(1+\eta)^{4}\left(1+\frac{4 \eta(1+m)^{3}}{m(1-\eta)}\right)^{2} .
\end{gathered}
$$

Thus, with $m>0$ fixed and small enough, we can choose $\eta>0$ small enough to satisfy (32).

\section{REFERENCES}

1. R. BARTNIK, Existence of maximal surfaces in asymptotically flat spacetimes, Comm. Math. Phys. 94 (1984) No. 2, 155-175.

2. H. BRAY, Proof of the Riemannian Penrose conjecture using the positive mass theorem, J. Differential Geom. 59 (2001), 177-267.

3. A. Chaljub-Simon And Y. Choquet-Bruhat, Problèmes elliptiques du second ordre sur une variété euclidienne à l'infini, Ann. Fac. Sci. Toulouse Math. (5) 1 (1979), No. 1, 9-25.

4. G. W. GibBons, The Isoperimetric and Bogomolny Inequalities for Black Holes, in Global Riemannian Geometry, edited by T. J. Willmore And N. Hitchin, John Wiley \& Sons, New York, 1984.

5. D. Gilbarg; N. S. Truginger, Elliptic Partial Differential Equations of Second Order Second Edition, Spriger-Verlag, New York 1983.

6. G. Huisken and T. Ilmanen, The Inverse Mean Curvature Flow and the Riemannian Penrose Inequality, J. Differential Geom. 59 (2001), 353-437.

7. J. Isenberg, R. Mazzeo And D. Pollack, Gluing and Wormholes for the Einstein Constraint Equations, Commun. Math. Phys. 231 (2002) 529-568.

8. J. Isenberg, N. Ó Murchadha, and J. W. York, Jr., Initial-Value Problem of General Relativity. III. Coupled Fields and the Scalar-Tensor Theory, Phys. Rev. D 13 No. 6 (1976), 1532-1537.

9. P. S. JANG, Note on Cosmic Censorship, Phys. Rev. D. 20 (1979) No. 4, 834-838.

10. J. M. Lee And T. H. PARker, The Yamabe Problem, Bull. of the AMS 17 No. 1 (1987) 37-92.

11. W. Meeks III, L. Simon, And S. T. Yau, Embedded minimal surfaces, exotic spheres, and manifolds with positive Ricci curvature, Ann. of Math. 16 (1982) No. 3, 621-659.

12. R. Schoen And S. T. YAU, On the proof of the positive mass conjecture in general relativity, Comm. Math. Phys. 65 (1979) No. 1, 45-76.

13. B. Smith And G. Weinstein, Quasiconvex foliations and asymptotically flat metrics of non-negative scalar curvature, Comm. Anal. Geom. 12 (2004) No. 3, 511-551.

14. E. Witten, A new proof of the positive energy theorem, Comm. Math. Phys. 80 (1981) No. 3, 381-402.

Department of Mathematics, University of Alabama at Birmingham, BirmINGHAm, Alabama 35205

E-mail address: weinstein@uab.edu

Mathematical Institute, Tohoku University, Sendai, Japan 980-8578

E-mail address: yamada@math.tohoku.ac.jp 